\title{
Comment on: Evans JM, Ogston SA, Emslie-Smith A, Morris A (2006) Risk of mortality and adverse cardiovascular outcomes in type 2 diabetes: a comparison of patients treated with sulfonylureas and metformin. Diabetologia 49:930-936
}

\author{
J. A. Johnson • S. H. Simpson • D. T. Eurich • \\ S. R. Majumdar
}

Received: 6 April 2006 /Accepted: 19 April 2006/Published online: 21 June 2006

(C) Springer-Verlag 2006

To the Editor: The recent paper by Evans et al. [1] on the risk of adverse cardiovascular outcomes in patients with type 2 diabetes treated with sulfonylureas or metformin is of great interest to us. The authors cited a study by our group [2], as having 're-ignited' this long-standing debate.

We found the similarity in risk estimates between the two studies striking, with both reporting a $40 \%$ lower mortality for metformin users compared to sulfonylurea users. This was particularly reassuring for us, as we did not have access to clinical data in the administrative databases we used [2]. Though Evans et al. did not include it in their cited literature, we also reported a follow-up analysis that evaluated non-fatal events defined by cardiovascular-related hospitalisations [3]. Interestingly, the results from Evans et al. were again very similar to ours, despite the lack of clinical data, with the risk of cardiovascular hospitalisation not increased for sulfonylurea users, suggesting similar baseline risk. An increased risk of hospitalisation was observed by Evans et al. for their Combination 2

\footnotetext{
J. A. Johnson $(\bowtie) \cdot$ D. T. Eurich $\cdot$ S. R. Majumdar

Faculty of Medicine and Dentistry, University of Alberta,

1200-10405 Jasper Avenue,

Edmonton, AB T5J 3N4, Canada

e-mail: jeff.johnson@ualberta.ca
}

J. A. Johnson · S. H. Simpson • D. T. Eurich · S. R. Majumdar Institute of Health Economics,

Edmonton, AB, Canada

\section{S. H. Simpson}

Faculty of Pharmacy \& Pharmaceutical Sciences,

University of Alberta,

Edmonton, AB, Canada (sulfonylurea first, metformin added) cohort, although, to our reading, it appears that their monotherapy and combination therapy cohorts were not entirely mutually exclusive (i.e. subjects in the Combination 1 and 2 cohorts also contributed time to the respective monotherapy cohorts), confounding their analyses with respect to duration and severity of diabetes.

Evans et al. suggest that these results support the hypothesis that metformin is cardioprotective, as did Sasali and Leahy [4] in an editorial accompanying our original paper. We question this conclusion, as it remains difficult to fully discern the causal relationship from this series of observational studies. We do recognise that an accumulation of evidence from observational studies can support inferences of causality. To that end, we recently conducted a dose-response relationship analysis within our study cohorts, and observed that increased exposure to sulfonylureas (based on either dosing or adherence) was associated with increased overall and cardiovascular-related mortality [5]. The relationship was particularly evident for the firstgeneration sulfonylureas, chlorpropamide and tolbutamide, but also apparent for glyburide. In contrast, we observed no difference in mortality associated with higher or lower doses of metformin. These results, along with a potential pathophysiological mechanism of impaired myocardial ischaemic preconditioning, prompted D. S. Bell in an accompanying editorial [6] to suggest that it is indeed sulfonylureas that are cardiotoxic.

Regardless of the pathophysiological explanation, we believe that the totality of available evidence, from trials [7] and observational studies [1-3,5], strongly supports the use of metformin as first-line therapy in type 2 diabetes. In the absence of randomised trial evidence of clinical benefit, 
older sulfonylureas should be relegated to third- or fourthline therapy for patients with type 2 diabetes.

\section{References}

1. Evans JM, Ogston SA, Emslie-Smith A, Morris A (2006) Risk of mortality and adverse cardiovascular outcomes in type 2 diabetes: a comparison of patients treated with sulfonylureas and metformin. Diabetologia 49:930-936

2. Johnson JA, Majumdar SR, Simpson SH, Toth EL (2002) Decreased mortality associated with the use of metformin compared with sulfonylurea monotherapy in type 2 diabetes. Diabetes Care 25:2244-2248
3. Johnson JA, Simpson SH, Toth EL, Majumdar SR (2005) Reduced cardiovascular morbidity and mortality associated with metformin use in subjects with type 2 diabetes. Diabet Med 22:497-502

4. Sasali A, Leahy JL (2002) Is metformin cardioprotective? Diabetes Care 26:243-244

5. Simpson SH, Majumdar SR, Tsuyuki RT, Eurich DT, Johnson JA (2006) Dose-response relationship between sulfonylureas and mortality in type 2 diabetes: a population-based cohort study. CMAJ 174:169-174

6. Bell DS (2006) Do sulfonylurea drugs increase the risk of cardiac events? CMAJ 174:185-186

7. UK Prospective Diabetes Study (UKPDS) Group (1998) Effect of intensive blood-glucose control with metformin on complications in overweight patients with type 2 diabetes (UKPDS 34). Lancet 352:854-865 